\title{
基于地形限制特征的泾河流域遥感地表覆被分类
}

\author{
洪 军 1,2 葛剑平 $2 *$ 蔡体久 2,3 聂忆黄4 \\ （1 农业部全国畜牧兽医总站, 北京 100026）（2 北京师范大学生态研究所, 生物多样性与生态工程重点实验室, 北京 100875）
}

（3 东北林业大学林学院,哈尔滨 150040）（4 中国环境科学研究院,北京 100012）

摘 要 由于在分类方法和空间分辨率等方面存在局限性, 基于粗分辨率遥感数据的传统非监督分类结果在不同 地物过渡带内往往误差较大。该文提出了基于地形限制特征的分类方法,在非监督分类的基础上,将非监督分类 结果按照像元进行细分, 并运用地形限制条件对细分后的像元进行二次判别分类。结果表明, 分类精度明显提高, 其中, 农田和居民点分类精度的提高最为明显。这一方法使得完全同质的单元可以进行属性的变更, 改善了像元 空间分辨率差造成的误差; 而地形限制特征的引入减少了传统非监督分类的不确定性,使模糊区域的分类有了较 为明确的区分特征, 提高了分类的精度。

关键词 像元细分 地形限制特征 遥感植被分类 泾河流域

\section{LAND COVER CLASSIFICATION OF REMOTELY SENSED IMAGERY USING A METHOD BASED ON TOPOGRAPHICAL RESTRICTIVE FEATURES: A CASE STUDY OF THE JINHE WATERSHED}

\author{
HONG Jun ${ }^{1,2}$ GE Jian-Ping ${ }^{2 *}$ CAI Ti-Jiu ${ }^{2,3}$ and NIE Yi-Huang ${ }^{4}$ \\ (1 China Animal Husbandry \& Veterinary Headstation, Beijing 100026, China) \\ (2 Ministry of Education Key Laboratory for Biodiversity Science and Ecological Engineering \& Institute of \\ Ecology, Beijing Normal University, Beijing 100875, China) \\ (3 College of Forestry, North-East Forest University, Harbin 150040, China) \\ (4 Chinese Research Academy of Environment Science, Beijing 100012, China)
}

\begin{abstract}
Based on non-supervised classification methods, multi-temporal images, such as NOAA-AVHRR, SPOT-VEGETATION and MODIS series data, have been used to map regional, continental or global land cover patterns. Because of limitations of the classification method and spatial resolution of images, traditional nonsupervised classification often results in many errors in the transition zones in which the spatial distribution of vegetation show segmental patterns. This problem can decrease the integral accuracy of the classification to a certain extent.
\end{abstract}

In this paper, a new method, the topographical restrictive features, is presented to classify remotely sensed images using the Jinhe watershed in the Loess plateau as a case study. First, 25 relatively independent classes were obtained using the non-supervised classification method with multi-temporal NDVI data derived from the first two bands of MODIS data. Every cell with $500 \mathrm{~m}$ spatial resolution of the classification determined by the non-supervised method was divided into 25 homogenous cells with $100 \mathrm{~m}$ spatial resolution. Then, topographical features were defined. Information about aspect, slope, elevation, river net structure, and vegetation regional characteristics, derived from 1:250 000 geographical spatial data, ETM + image and yield data, were used to construct topographical restrictive features. Finally, the classification of every sub-cell was tested using the restrictive features and some cells were reclassified while maintaining their original classification. After the secondary classification, all cells were labeled by land cover type according to the land cover classification system, which was defined previously on the base of the IGBP land cover classification system and UMD system.

Using this new method, the accuracy of the land cover classification increased from $71.88 \%$, in traditional non-supervised classification method, to $84.09 \%$. In particular, the accuracy of cropland and urban type classification improved. The method of cell sub-division avoids the shortcomings of traditional classification methods owing to the coarse resolution of the image processing, and makes it more highly probable that land cover types are homogenous within cells. The introduction of topographical restrictive features decreases 
uncertainty of traditional fuzzy classification and provides more precise distinctive features to classify the fuzzy zone, and thus improves the accuracy of classification.

Key words Image segmentation, Topographical restrictive features, Remotely sensed land cover classification, Jinhe watershed

随着遥感技术的发展, 人类可以更好地揭示、描 绘和刻划地球表面覆被特征, 更加深入地了解地球 表层发生的各种现象和过程。虽然近年来新的遥感 分类方法不断出现，如模糊集 (Marselli et al .,1996)、 神经网络分类器 (Foody, 1999; Skidmore et al., 1997)、 空间逐步寻优模型(周成虎等, 2001)、分层聚类(骆 剑承等, 1999)等,在影像分类精度等方面有了不少 的改进,但由于这些方法上多集中在利用高分辨率 的遥感影像进行的小尺度案例研究上, 对于采用多 时相遥感数据为信息源的区域、洲际和全球尺度的 研究很难加以应用。多时相数据往往具有高时间分 辨率、低空间分辨率的特点,对于地面景观较为破碎 的区域,其像元特征是地表多特征的空间集合,在分 类结果中往往是各种类型的混合体, 给分类带来了 一定的难度。大量的研究表明, 辅助数据 (如高程数 据、降水量、温度、土壤数据等) 参与分类, 可以提高 分类的精度 (陈云浩等, 2001, 2002; 陈玉敏, 2002)。 目前, 在景观和群落尺度的植被格局分析中,地形因 素的作用已经引起关注 (Kikuchi, 1990; Kikuchi \& Miura, 1993; Pinder et al., 1997; 沈泽吴等,2000)。 本植被生态学家以不同的方式进行地形单元分类和 赋值, 对亚热带森林群落、物种及干扰的分布格局和 地形的相关关系做了大量的研究工作, 从理论上分 析了地形对生态系统格局和过程产生影响的各个方 面 (Hara et al., 1996; Swansond et al ., 1988)。Ostendorf 和 Reynold( 1998) 根据阿拉斯加北部山地植被格 局受地表径流深的控制, 利用 GIS 从数字高程模型 (DEM)中生成坡度分布图和泾流分布图, 建立了一 个基于地形的植被格局模型。Brigitt (2002) 证明地 形是促使植被格局形成的一个重要因子。

本文以泾河流域为例, 在传统非监督分类的基 础上, 将非监督分类的结果按照像元细分, 利用设定 地形限制条件对细分后的像元进行二次分类, 以达 到提高分类精度的目的。

\section{1 试验数据与研究方法}

\section{1 研究区域选择}

试验区位于地处陕甘宁交界处的泾河流域。泾 河流域 $\left(106^{\circ} 20^{\prime} \sim 108^{\circ} 48^{\prime} \mathrm{E}, 34^{\circ} 24^{\prime} \sim 37^{\circ} 20^{\prime} \mathrm{N}\right)$ 位于
黄土高原中部, 处于六盘山和子午岭之间, 流域绝大 部分属于陇东黄土高原, 是渭河流域的最大支流, 黄 河的二级支流。流域总面积约 $45421 \mathrm{~km}^{2}$, 是黄河 中游四大支流无定河、渭河、泾河和北洛河中来沙量 最多的一条支流。

\section{2 试验数据选择与预处理}

数据源选用全球土地利用数据库组织 (Global land cover facility, GLCF) 提供的欧亚大陆 $32 \mathrm{~d}$ 组合 中分辨率成像光谱仪 (Moderate resolution imaging spectroradiometer, MODIS) 1、2 波段数据, 空间分辨率 为 $500 \mathrm{~m}$ 。通过比值法获取归一化差异植被指数 (Normalized difference vegetation index, NDVI), 2002 年 1 月 2003 年 1 月多时相 $N D V I$ 数据将作为分类的 基础数据。

在分类系统上, 本研究在国际地圈-生物圈计划 (International geosphere-biosphere programme, IGBP) 和 马里兰大学 (University of Maryland, UMD) 全球土地覆 被分类系统的基础上, 针对泾河流域, 构建了一个新 的地表覆被分类系统。分类系统的类型、编码、定义 及描述见表 1 。

\section{3 研究方法}

首先对多时相数据进行非监督分类, 将研究区 域的地表覆被进行初步分类, 然后利用野外调查资 料、1: 25 万地理空间数据、ETM 数据( 1999 2002 年, 计 9 景), 建立地形限制特征, 根据这些特征或者 特征组合, 将初步分类结果按照 $1: 25$ 万数据细分为 同质的单元，继而根据设定的地形限制特征或者特 征组合识别地表覆被类型, 从而完成分类, 最后对分 类精度进行评价。具体过程见图 1 所示:

\subsection{1 遥感数据分类及像元细分}

遥感影像初步分类采用非监督分类。非监督分 类只是根据遥感数据本身多时相性所包含的植被季 相特征的差异, 将多维空间特征相似的点聚焦成一 个“点群”, 每个 “点群”形成一个相对独立的类型。 这些类型只是对像元进行了区分, 并不确定各类的 属性, 分类后必须进行后处理, 以确定类型及其属 性。受计算方法和空间分辨率的限制, 非监督分类 的一个最大缺点就是分类后同物异谱、同谱异物现 象明显, 分类精度往往较低, 特别是在植被类型较为 
表 1 本研究采用的土地覆被分类系统编码及描述

Table 1 Code and description of land cover classification system in research project

\begin{tabular}{|c|c|c|}
\hline $\begin{array}{l}\text { 类型 } \\
\text { Type }\end{array}$ & $\begin{array}{l}\text { 编码 } \\
\text { Code }\end{array}$ & $\begin{array}{c}\text { 描述 } \\
\text { Description }\end{array}$ \\
\hline 森林 & 1 & 主要覆被类型为林木, 冠层盖度大于 60\%, 树高大于 5 m \\
\hline Forest & 1 & Dominated by trees, and canopy cover is more than $60 \%$ and height exceeds $5 \mathrm{~m}$ \\
\hline 矮林、郁闭灌从 & & $\begin{array}{l}\text { 主要覆被类型为矮林和灌木, 冠层盖度大于 } 40 \% \text {, 高度不超过 } 5 \mathrm{~m} \text {, 地表裸露或为草被。注: 本研究中果 } \\
\text { 树划分为此类更为合适 }\end{array}$ \\
\hline $\begin{array}{l}\text { Closed bushland or } \\
\text { shrubland }\end{array}$ & 2 & $\begin{array}{l}\text { Dominated by bush or shrub, and canopy cover is more than } 40 \% \text { and height does not exceed } 5 \mathrm{~m} \text {. The remaining } \\
\text { cover is the barren or herbaceous. Note: Fruit tree is classified as the appropriate bushland or shrubland in this study }\end{array}$ \\
\hline $\begin{array}{l}\text { 稀疏灌从 } \\
\text { Opened shrubland }\end{array}$ & 3 & $\begin{array}{l}\text { 主要覆被类型为灌丛,冠层盖度大于 } 10 \% \text {, 小于 } 40 \% \text { 。灌木高度不超过 } 2 \mathrm{~m} \text {, 地表裸露或者为草被 } \\
\text { Dominated by shrub, and canopy cover is more than } 10 \% \text { and less than } 40 \% \text {. Shrubs do not exceed } 2 \mathrm{~m} \text { in height. } \\
\text { The remaining cover is the barren or herbaceous }\end{array}$ \\
\hline 农田 & & 景观中以农业用地为主,农用地比例大于 80\% \\
\hline Cropland & 4 & Dominated by crop-producing fields, which covered more than $80 \%$ \\
\hline $\begin{array}{l}\text { 高盖度草地 } \\
\text { High density grassland }\end{array}$ & 5 & $\begin{array}{l}\text { 主要类型为草被, 灌木冠层盖度小于 } 10 \% \text {, 草本盖度大于 } 60 \% \\
\text { Dominated by continuous herb, and less than } 10 \% \text { covered by tree or shrub and more than } 60 \% \text { covered by continu- } \\
\text { ous herb }\end{array}$ \\
\hline 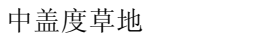 & & 主要类型为草被,灌木冠层盖度小于 10\%,草本盖度大于 30\%,小于 60\% \\
\hline $\begin{array}{l}\text { Medium density grass- } \\
\text { land }\end{array}$ & 6 & $\begin{array}{l}\text { Dominated by herb, and less than } 10 \% \text { covered by tree or shrub, more than } 30 \% \text { and less than } 60 \% \text { covered by } \\
\text { herb }\end{array}$ \\
\hline $\begin{array}{l}\text { 低盖度草地 } \\
\text { Low density grassland }\end{array}$ & 7 & $\begin{array}{l}\text { 主要类型为草被, 灌木冠层盖度小于 } 10 \% \text {, 草本盖度小于 } 30 \% \text {, 地表无沙化现象 } \\
\text { Dominated by herb, and less than } 10 \% \text { covered by tree or shrub and less than } 30 \% \text { covered by herb. There is no de- } \\
\text { sertification on the land surface }\end{array}$ \\
\hline $\begin{array}{l}\text { 半荒漠草地 } \\
\text { Semi-desert grassland }\end{array}$ & 8 & $\begin{array}{l}\text { 主要类型为草被, 灌木冠层盖度小于 } 10 \% \text {, 草本盖度小于 } 10 \% \text {,地表出现沙化现象 } \\
\text { Dominated by herb, and less than } 10 \% \text { covered by tree or shrub and less than } 10 \% \text { covered by herb. There is deser- } \\
\text { tification on land surface at local site }\end{array}$ \\
\hline $\begin{array}{l}\text { 城镇 } \\
\text { Urban and built-up }\end{array}$ & 9 & $\begin{array}{l}\text { 主要类型为建筑物和其它人为建筑 } \\
\text { Covered by building or other man-made structure }\end{array}$ \\
\hline
\end{tabular}

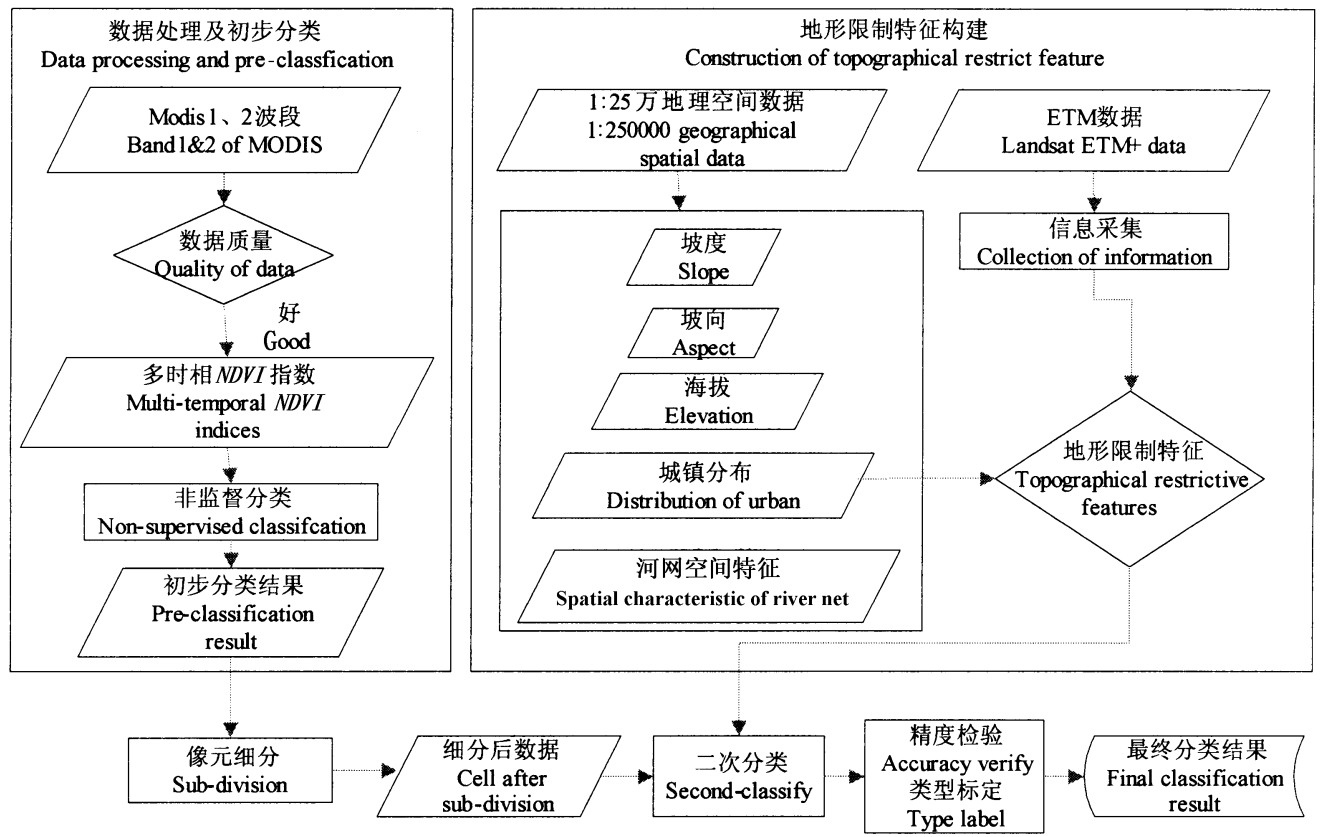

Fig.1 Vegetation remotely sensed classification based on topographical restrictive features 
复杂的区域和过渡带更为明显。

在本研究的初步分类中, 将研究区域的地表覆 被类型区分为 25 种类型, 这些类型间存在着重合 (即 2 种或多种类型同属于一种地物) 与交叉 (即 1 种类型属于两种地物); 前者可以通过后分类处理的 标定工作实现,对于后者,本研究采用将像元细分后 通过设定地理限制条件的方法来实现。具体做法是 将非监督的分类结果按照 $1: 25$ 万空间数据标准进 行细分, 将分辨率为 $500 \mathrm{~m}$ 的非监督分类结果细分 为 $100 \mathrm{~m}$ 空间分辨率的同属性单元, 然后利用地形 特征限制条件对细分的单元进行二次分类，以达到 提高分类精度的目的。像元细分及二次分类过程见 图 2 所示:

\subsection{2 地形限制特征的构建}

本文利用 $1: 25$ 万地理空间数据, 通过 GIS 手段 获取泾河流域坡度、坡向、海拔、河网分布特征等信 息。25旧界坡度(根据国家天然林保护工程, > $25^{\circ}$ 的坡耕地必须还林或者还草。根据两次考察，该区 域 $>25^{\circ}$ 的坡耕地基本已经实现还草。、、阴阳坡植被 差异(阳坡中低盖度草地出现的概率要明显高于高 盖度草地的概率, 阴坡相反)、植被分布海拔梯度特 征、河网周围植被分布特征(河网周围一定范围是农 田出现的高频率地带)等信息将会作为构建地形限 制条件的信息源; 利用 1999 2002 年覆盖全流域的 $\mathrm{ETM}+$ 数据, 通过人机交互式解译, 获取农业区、草 地、森林、城镇等易目视解译的地物的大致分布范 围, 这种人机交互式解译可以获取较为准确的农田、 森林、城镇的分布范围，可作为判断这些类型分布的 限制特征; 2003、2004 年先后两次前往泾河流域进行 实地考察, 沿途设置调查样点和样带, 记录地表覆被
基本状况, 形成第一手的辅助分类的限制条件, 这些 资料将有助于判断混淆类型的归属问题。

\subsection{3 二次分类及像元属性判别}

本研究非监督分类将研究区域的地表类型划定 为 25 种类型, 第 1 类可以初步判断为半荒漠草原或 者城镇类型, 第 25 类可以初步判断为森林, 整个分 类结果呈半荒漠草原、城镇-草地-农田-灌丛-森林梯 度排列, 类型的混淆往往发生在两种地物发生突变 的过渡区域。传统的非监督方法的分类后标定通常 是将相邻的类型归并为一类, 在不同地物的过渡区 域往往存在着混合类型, 传统的非监督分类通常将 这一类型划定为混合类型, 模糊了分类的界限。将 非监督分类结果按照像元进行细分, 使得混合类型 能够得到二次分类的机会; 利用地形限制对混合类 型进行标定，清晰了传统分类方法对混合像元分类 的模糊界限。

适宜的地形限制特征的构建则是保证二次分类 结果准确性的基础。二次分类主要是根据地形限制 特征, 将非监督分类方法中出现的交叉现象 (即 1 种 类型属于两种地物) 通过像元细分和二次分类的方 法实现准确划分与标定。例如: 在农田与草地混淆 的地带, 坡度 $>25^{\circ}$ 的区域基本可以判定为草地, 坡 度 $<10^{\circ}$ 的区域基本可以判定为农田; 在农田与灌从 混淆的地带, 坡度 $>25^{\circ}$ 的像元基本可以判定为灌 丛; 落入农业区的森林类型基本可以判断为灌从类 型 (果树); 阳坡草本为中低密度草地的概率明显高 于高盖度草地出现的概率, 辅以区域海拔梯度特征, 能较好区分 3 种不同盖度的草地; 落入 ETM + 解译 城镇范围的非监督分类第 1 类基本可以判定为城镇 等。

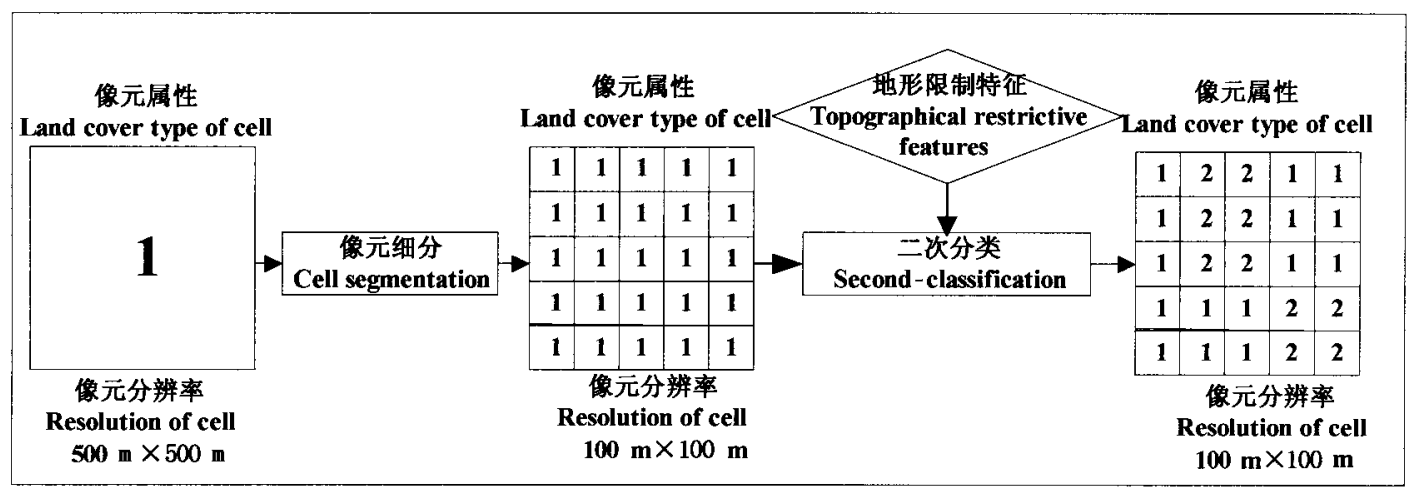

图 2 像元细分及子像元二次分类

Fig.2 Subdivision and second-classification of image cell 
图 3 两种分类结果对比(局部)

Fig.3 Comparison of two classification results (local area showed) 


\section{2 结果与讨论}

\section{1 两种不同分类方法精度检验}

为了验证不同分类结果的精度, 本研究根据 $\mathrm{ETM}+$ 数据和野外调查数据, 随机抽取 352 个像元, 两种不同分类方法的精度见表 2。传统非监督分类 将预分的 25 类通过类组合合并为本文定义的 9 种 基本地表覆被类型。

\section{2 结果比较与分析}

由表 2 可以看出, 基于地形限制特征的分类精 度较传统的非监督分类有了较大的改善。传统的非 监督分类是以建立像元光谱特征的统计分析为基 础, 限于方法本身的局限性和空间分辨率, 在不同地 物过渡带内往往误差较大(图 2)。通过将非监督分
类结果细分, 同属性区域首先被建立, 然后将地形限 制条件运用于这些同属性子像元上, 进行二次判别 分类, 各种地物类型的分类精度都有了较大的改善。 这种基于地形限制特征的子像元二次分类的精度要 明显要高于传统的基于像元层次的非监督分类。其 中, 农田和居民点分类精度的提高最为明显。在分 类中, 地形限制特征的选择过程也是各种领域知识、 专家经验和规则融入分类的过程, 从而使基于地形 限制特征的分类更加具有智能性。像元细分使得完 全同质的单元可以进行属性的变更, 改变了基于像 元分类的局限性; 而地形限制特征的引入在减少遥 感分类不确定性的同时, 也提高了分类的精度。整 个流域的分类结果见图 3。

表 2 两种不同方法下的分类精度报告

Table 2 Classification accuracy report based on two different classification methods

\begin{tabular}{|c|c|c|c|c|c|}
\hline \multirow{2}{*}{$\begin{array}{c}\text { 类名 } \\
\text { Type code }\end{array}$} & \multirow{2}{*}{$\begin{array}{l}\text { 参考像元 } \\
\text { Referred cell }\end{array}$} & \multicolumn{2}{|c|}{$\begin{array}{c}\text { 非监督分类 } \\
\text { Non-supervised classification }\end{array}$} & \multicolumn{2}{|c|}{$\begin{array}{c}\text { 基于地理限制特征的分类 } \\
\text { Topographical restrictive features classification }\end{array}$} \\
\hline & & $\begin{array}{c}\text { 正确分类像元 } \\
\text { Acutely classified cell }\end{array}$ & $\begin{array}{c}\text { 精度 } \\
\text { Accuracy }(\%)\end{array}$ & $\begin{array}{c}\text { 正确分类像元 } \\
\text { Acutely classified cell }\end{array}$ & $\begin{array}{c}\text { 精度 } \\
\text { Accuracy }(\%)\end{array}$ \\
\hline 1 & 54 & 42 & $77.78 \%$ & 46 & $85.19 \%$ \\
\hline 2 & 42 & 30 & $71.43 \%$ & 35 & $83.33 \%$ \\
\hline 3 & 40 & 28 & $70.00 \%$ & 34 & $85.00 \%$ \\
\hline 4 & 68 & 44 & $64.71 \%$ & 58 & $85.29 \%$ \\
\hline 5 & 36 & 25 & $69.44 \%$ & 30 & $83.33 \%$ \\
\hline 6 & 32 & 25 & $78.13 \%$ & 28 & $87.50 \%$ \\
\hline 7 & 40 & 31 & $77.50 \%$ & 34 & $85.00 \%$ \\
\hline 8 & 28 & 21 & $75.00 \%$ & 23 & $82.14 \%$ \\
\hline 9 & 12 & 7 & $58.33 \%$ & 10 & $83.33 \%$ \\
\hline 合计 Sum & 352 & $\begin{array}{l}253 \\
\text { 分类精度 Accl }\end{array}$ & $.88 \%$ & $\begin{array}{l}296 \\
\text { 分类精度 Accul }\end{array}$ & $09 \%$ \\
\hline
\end{tabular}

\section{3 结论与讨论}

本文在传统非监督分类的基础上,提出将非监 督分类结果细化, 基于地形限制特征的植被遥感分 类方法。该方法在分类精度上较传统非监督分类有 了较大的改善,地形限制特征的引入只是一个初步 的尝试, 在今后的工作中我们将会尝试多特征用于 粗分辨率数据的分类。

在本文研究中亦发现,像元的细分在一定程度 上加大了研究区域地表覆被遥感分类结果的破碎化 程度。如何确定适合空间尺度，在保持分类精度的 同时降低分类结果的破碎化程度亦是今后分类中必 须面对的一个问题。

\section{参 考 文 献}

Brigitte D (2002) . Landscape pattern in topographically complex landscapes: issues and techniques for analysis. Landscape Ecology, 17, $729-743$.

Chen YH(陈云浩), Li XB(李晓兵), Shi PJ (史培军) (2001). Variation in NDVI driven by climate factors across China, 1983 - 1992. Acta Phytoecologica Sinica (植物生态学报), 25, 716 - 720. (in Chinese with English abstract)

Chen YH(陈云浩), Li XB(李晓兵), Shi PJ(史培军) (2002). Landscape spatial-temporal pattern analysis on change in the fraction of green vegetation based on remotely sensed data: a case study in Haidian District, Beijing. Acta Ecologica Sinica (生态 学报), 22, 1581 - 1586. (in Chinese with English abstract)

Chen YM(陈玉敏) (2002). Application of neural network to classification of remote sensing image. Journal of Geomatics (测绘信 
息与工程) $, 27(3), 6-8$. (in Chinese with English abstract)

Hara M, Hirata K, Oono K (1996) . Relationship between microlandform and vegetation structure in an evergreen broad-leaved forest on Okinawa Island, S-W, Japan. Natural History Research, 4, $27-35$.

Foody G (1999). Image classification with neural network: from completely-crisp to fully fuzzy dituations. In: Atkinson PM, Tate NJ eds. Advances in Remote Sensing and GIS Analysis. Wiley, Chichester, New York, $17-37$.

Kikuchi T (1990). A DCA analysis of floristic variation of plant communities in relation to microland from variation in hillshade area. Ecological Review, 22, 25 - 31 .

Kikuchi T, Miura O (1993). Vegetation patterns in relation to micro-scale landforms in hilly land regions. Vegetatio, 106, 147 154 .

Luo JC(骆剑承), Leng Y (梁怡), Zhou CH(周成虎)（1999）。 Scale space based hierarchical clustering method and its application to remotely sensed data classification. Acta Geodaetica et Cartographica Sinica (测绘学报), 28, 319 - 324. (in Chinese with English abstract)

Marselli F, Rudolf A, Cons CE (1996) . Fuzzy classification of spatially degraded thematic mapper data for the estimation of sub-pixel components. International Journal of Remote Sensing, 17, 537
-551 .

Ostendorf B, Reynold JF (1998). A model of arctic tundra vegetation derived from topographic gradients. Landscape Ecology, 13, $187-202$.

Pinder JE III, Kroh GC, White JD, Basham MAM (1997). The relationships between vegetation types and topography in Lassen Vocalnic National Park. Plant Ecology, 131, 17 - 29.

Shen ZH (沈泽昊), Zhang XS (张新时), Jin YX (金义兴). An analysis of the topographical patterns of the chief wooly species at Dalaoling in the three Gorges Kegion. Acta Phytoecologica Sinica (植物生态学报), 24, 581 - 587. (in Chinese with English abstract)

Skidmore A, Tumer B, Brinkbof W, Knowles E (1997). Performance of neural network: mapping forest using GIS and remotely sensed data. ISPRS Journal of Photogrammetric Engineering \& Remote Sensing, 63, $501-514$.

Swansond FJ, Kratz TK, Caine N (1988). Landform effects on ecosystem patterns and process. BioScience, 38, $92-98$.

Zhou CH(周成虎), Luo JC(骆剑承), Yang CJ(杨存建), Liu QS(刘庆生) (2001). Geographical Understanding and Analyses of Remote Sensed Imagery (遥感影响像地学理解与分析). Science Press, Beijing. (in Chinese) 